\title{
Pseudogout of the cervical and thoracic spine mimicking infection after lumbar fusion: case report
}

\author{
Kelly J. Bridges, MD, ${ }^{1}$ Carli L. Bullis, MD, ${ }^{1}$ Ajay Wanchu, MD, ${ }^{2}$ and Khoi D. Than, MD${ }^{1}$ \\ ${ }^{1}$ Department of Neurological Surgery, and ${ }^{2}$ Division of Arthritis \& Rheumatic Diseases, Department of Medicine, Oregon Health \& \\ Science University, Portland, Oregon \\ Pseudogout is a form of acute calcium pyrophosphate deposition (CPPD) disease that typically afflicts the elderly. CPPD \\ commonly involves larger joints, such as the knees, wrists, shoulders, and hips, and has been known to involve the \\ spine. \\ The authors report the case of a 66 -year-old woman with a recent history of lumbar laminectomy and fusion who \\ presented 5 weeks postprocedure with a clinical and radiographic picture consistent with multilevel skip lesions involving \\ the cervical and thoracic spine, thoracic discitis, and epidural abscess. Serial blood cultures and repeat biopsy samples \\ were sterile. Subsequent wrist and ankle erythema, pain, and swelling led to synovial fluid analysis, and pseudogout was \\ diagnosed. She was treated with an interleukin-1 inhibitor with immediate symptom relief. \\ To the authors' knowledge, this is only the second report of spinal pseudogout presenting with a clinical and radiographic \\ picture consistent with discitis and epidural abscess. This report is the first to report skip lesions of pseudogout occurring \\ throughout the spine that are uniquely remote from a recent lumbar surgery. \\ https://thejns.org/doi/abs/10.3171/2016.12.SPINE16979
}

KEY WORDS pseudogout; calcium pyrophosphate deposition; CPPD; cervical; lumbar; thoracic

$\mathrm{P}$ SEUDOGOUT is a widely recognized form of acute calcium pyrophosphate deposition (CPPD) disease, presenting as acute arthritis of monoarticular or oligoarticular joints. ${ }^{25}$ CPPD affects $4 \%-7 \%$ of adults in the United States. ${ }^{2,25}$ The disease typically afflicts people older than 60 years, with increasing incidence with age. ${ }^{27}$ Common signs include erythema, warmth, and swelling of the affected joint, with a clinical presentation similar to gout or septic arthritis. ${ }^{23}$ Patients often present with fevers, chills, and constitutional symptoms lasting weeks to months. ${ }^{25}$ Prior trauma to the joint is a strong risk factor, and acute attacks can occur in the setting of illness, joint trauma, or during the postoperative period. ${ }^{25}$

Pseudogout is characterized by the accumulation of calcium pyrophosphate dihydrate crystals in the articular and periarticular tissues, ${ }^{2,25}$ and pathological examination demonstrates positively birefringent rhomboid-shaped crystals in the synovial fluid of the affected joint.$^{25}$ Therapy is directed toward reducing inflammation and includes intraarticular glucocorticoids, systemic glucocorticoids, oral colchicine, and nonsteroidal antiinflammatory drugs. ${ }^{14}$

While pseudogout commonly affects the knees, wrists, shoulders, and hips, it has also been documented to involve the spine, most often the cervical spine, followed by the lumbar spine, and rarely the thoracic spine. ${ }^{27}$ When the atlantooccipital joint is involved (referred to as crowned dens syndrome), the condition results in acute cervicooccipital pain with fever, neck stiffness, and elevated inflammatory markers. ${ }^{7}$ Typical radiographic manifestations of CPPD in the spine include calcium deposition within the supraspinous and interspinous ligaments, anterior and posterior longitudinal ligaments, intervertebral discs, and joint capsule. ${ }^{21}$

Grobost et al. described a case of lumbar CPPD presenting with clinical and imaging findings concerning for septic spondylodiscitis and epidural abscess. ${ }^{8}$ Cultures were sterile, while pathology revealed calcium pyrophosphate crystals. The patient was treated with colchicine; symptomatic improvement was observed within 3 days and normalization of inflammatory markers within a week. To our knowledge, this is the only previously reported case of acute CPPD of the spine initially mistaken for discitis and epidural abscess.

Here, we report the case of a patient with similar findings. However, this particular case is unique in that the patient initially underwent lumbar laminectomies and fusion

ABBREVIATIONS CPPD = calcium pyrophosphate deposition.

SUBMITTED August 18, 2016. ACCEPTED December 30, 2016.

INCLUDE WHEN CITING Published online May 19, 2017; DOI: 10.3171/2016.12.SPINE16979. 
only to present 5 weeks later with skip lesions involving the cervical and thoracic spine, another feature of spinal pseudogout that, to our knowledge, has not been previously reported.

\section{Case Report}

History and Presentation

A 66-year-old woman presented to our spine clinic with longstanding, progressively worsening low-back pain as well as bilateral lower-extremity pain and paresthesias. On examination, the patient had difficulty with heel gait, which was presumed to be secondary to right tibialis anterior weakness, and sensation to light touch was subjectively decreased in her left lower extremity. Lumbar spine MRI revealed lumbar stenosis from L2 to L5 with L4-5 spondylolisthesis (Fig. 1). Scoliosis radiographs demonstrated an S-shaped thoracolumbar scoliosis, trace C4-5 anterolisthesis, and normal sagittal balance (Fig. 2). Previous conservative treatment consisting of physical therapy, gabapentin, and nonsteroidal antiinflammatory medications had failed. Her pain management specialist did not think that she was a candidate for epidural steroid injections given the extent of stenosis.

\section{Surgery}

The patient underwent L2-5 laminectomies, bilateral foraminotomies, and L4-5 transforaminal lumbar interbody fusion with L4-5 posterior instrumented fusion. She did well postoperatively with resolution of her bilateral lower-extremity pain and her paresthesias, as well as moderate improvement in her back pain.

\section{Postoperative Course}

Approximately 5 weeks after surgery, the patient presented to the emergency department with 2 days of midback spasms, fever, and chills. Her temperature was $102^{\circ} \mathrm{F}$, and blood inflammatory markers were elevated. Specifically, her erythrocyte sedimentation rate was $72 \mathrm{~mm} / \mathrm{hr}$ (reference range $0-30 \mathrm{~mm} / \mathrm{hr}$ ), while her C-reactive protein level was $96 \mathrm{mg} / \mathrm{L}$ (reference range $<10.0 \mathrm{mg} / \mathrm{L}$ ). Her

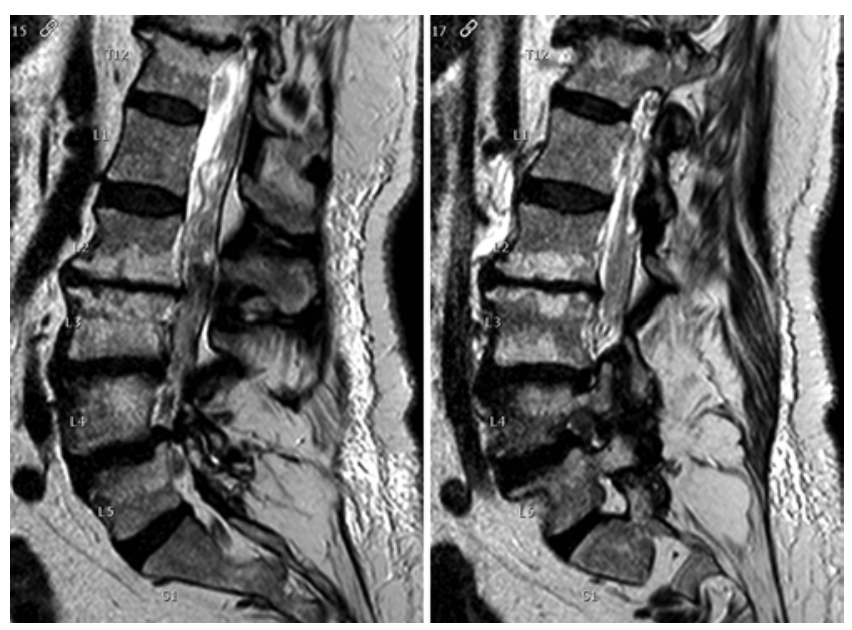

FIG. 1. Sagittal preoperative lumbar spine MR images demonstrating lumbar stenosis from L2 to L5 with L4-5 spondylolisthesis.

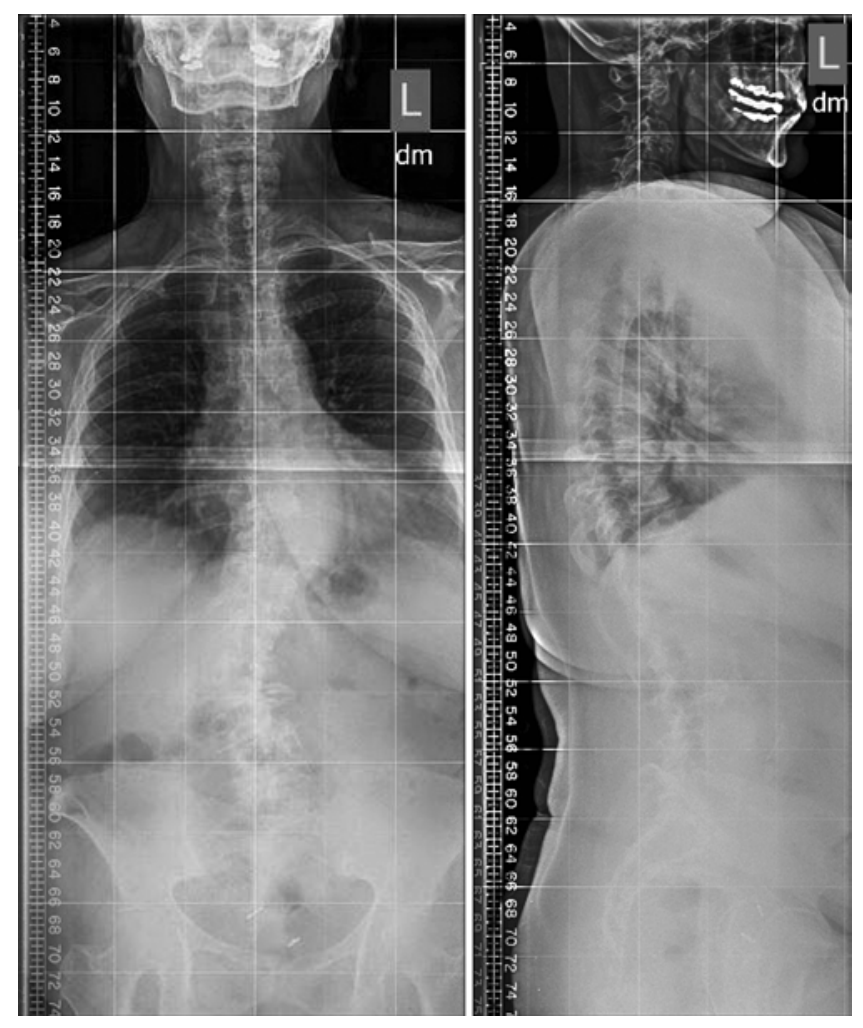

FIG. 2. Posteroanterior (left) and lateral (right) scoliosis radiographs demonstrating an S-shaped thoracolumbar scoliosis and normal sagittal balance.

white blood cell count was normal, and blood cultures were sterile. Thoracic and lumbar spine MRI demonstrated some contrast enhancement within the C7-T1 facet joints as well as the T1-2 and T9-12 intervertebral discs. There was also ventral epidural enhancement from $\mathrm{T} 9$ to T12 and some enhancement within the lumbar surgical site, presumed to be consistent with postoperative changes (Fig. 3). A CT-guided biopsy of the T11-12 disc space was performed, and cultures were sterile. Pathology revealed normal cartilage and bone without inflammation or malignancy. Our infectious diseases team was consulted, and a 6-week course of vancomycin and piperacillin-tazobactam, empirical treatment for discitis, was recommended.

The patient returned to the emergency department 2 weeks later with increased back pain and a fever of $102^{\circ} \mathrm{F}$. Inflammatory markers were even more elevated (erythrocyte sedimentation rate $91 \mathrm{~mm} / \mathrm{hr}$, and C-reactive protein $227 \mathrm{mg} / \mathrm{L}$ ). Full-spine MRI revealed marked increase in the enhancement of the C7-T1 facet joints with extension into the paraspinal soft tissues and the posterior epidural space, resulting in spinal cord compression (Fig. 4). The patient subsequently underwent a left C7-T1 laminotomy for biopsy sampling of the lamina, facet joint, ligamentum flavum, and epidural tissue. Tissue was sent to the microbiology department for $16 \mathrm{~S}$ ribosomal polymerase chain reaction testing in accordance with instructions from the infectious diseases team; this biopsy sample showed no evidence of infectious etiology. Histopathological examination revealed bone and fibroconnective tissue with reactive changes, predominantly a large number of mac- 

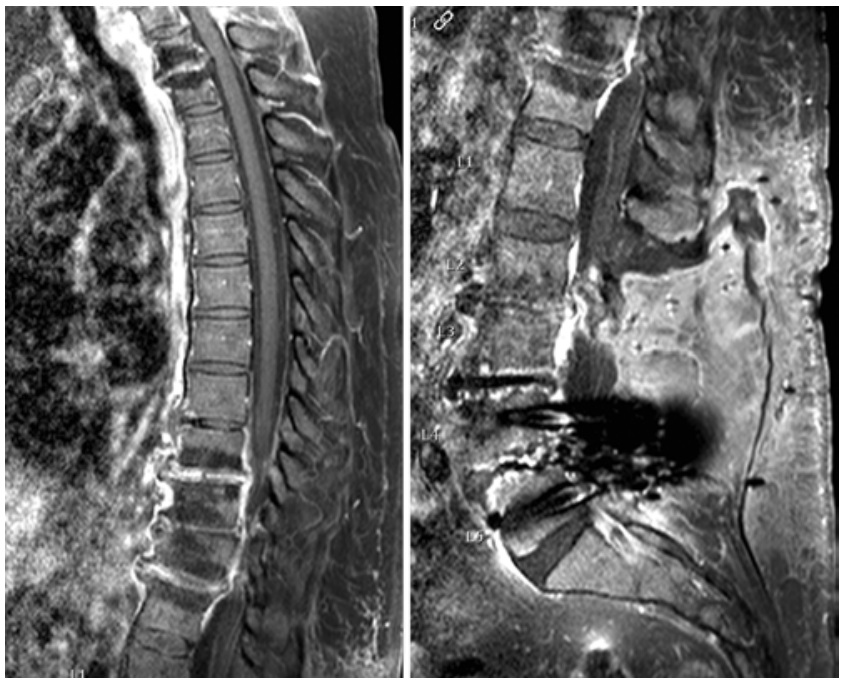

FIG. 3. Five weeks after lumbar surgery, thoracic (left) and lumbar (right) spine MR images demonstrate some enhancement within the C7-T1 facet joints as well as the T1-2 and T9-12 intervertebral discs. There is also ventral epidural enhancement from T9 to T12 and some enhancement within the lumbar surgical site.

rophages and reactive hyperplasia of the stroma (Fig. 5). On later review, there was additionally some material suggestive of pseudogout, although this was nonpolarizable. However, pseudogout was not considered in the differential diagnosis at the time of biopsy, and no further processing was performed. Serial blood cultures remained sterile.

One week later, the patient developed sudden onset of bilateral ankle and right wrist pain, and her joints were notably swollen and erythematous. Wrist radiography demonstrated chondrocalcinosis, which is a radiographic correlate of CPPD crystals. The rheumatology team was consulted and suggested that the patient's presentation and radiographic findings were consistent with a diagnosis of acute pseudogout. A joint aspiration of her left ankle was therefore performed, but was not sent for crystal examination. However, the rheumatologist (A.W.) identified some objects suspicious for calcium pyrophosphate deposition. The patient was treated with a 3-day course of anakinra (an interleukin-1 inhibitor), and all of her symptoms resolved. One month later, she was again seen in the spine clinic and reported resolution of her back symptoms and no further arthritis flairs. To date, she has been monitored closely by the rheumatology team and has been prescribed colchicine to be taken as needed. She has not undergone repeat spine MRI, and her serum inflammatory markers have not been reassessed, as she is clinically doing well at 7 months following the initial surgery.

\section{Discussion}

This report of pseudogout involving skip lesions of the cervical and thoracic spine following a lumbar fusion is unique. To our knowledge, earlier reported cases of pseudogout have involved focal, contiguous CPPD.

Acute CPPD disease, also known as pseudogout, affects $4 \%-7 \%$ of adults in the United States. ${ }^{2,6}$ There is increasing prevalence with age such that approximately $44 \%$
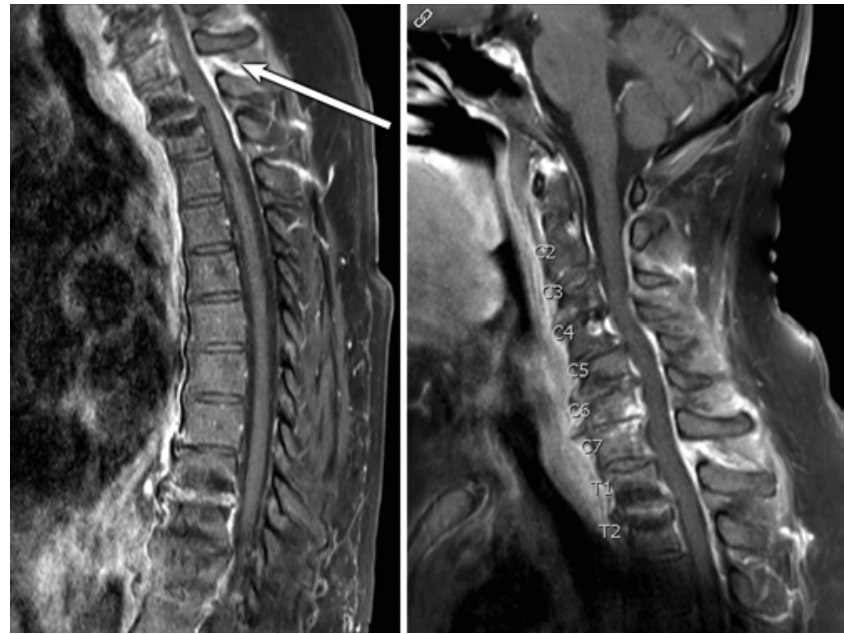

FIG. 4. Approximately 7 weeks after lumbar surgery, full-spine sagittal MR images demonstrate a marked increase in the enhancement of the C7-T1 facet joints with extension into the paraspinal soft tissues and the posterior epidural space, resulting in cord compression (arrow).

of people older than 84 years have CPPD. ${ }^{25}$ Furthermore, $66 \%$ of patients with chronic arthritis complaints related to CPPD will also have superimposed acute episodes. ${ }^{26}$

Patients with pseudogout often present with fevers, chills, and constitutional symptoms lasting weeks to months, and the clinical presentation can be similar to gout or septic arthritis. ${ }^{23,25}$ The disease typically affects larger joints with cardinal signs of inflammation including warmth, tenderness, erythema, and swelling. ${ }^{5}$ However, the spine can also be affected. Patients are often asymptomatic with incidental radiographic findings; however, clinical manifestations can result due to compression of the spinal cord and nerve roots..$^{15}$ Radiographic findings include disc space loss with adjacent vertebral body sclerosis, apophyseal joint abnormalities, and subluxation. ${ }^{28}$ However, unique to the case we present, radiographic find-

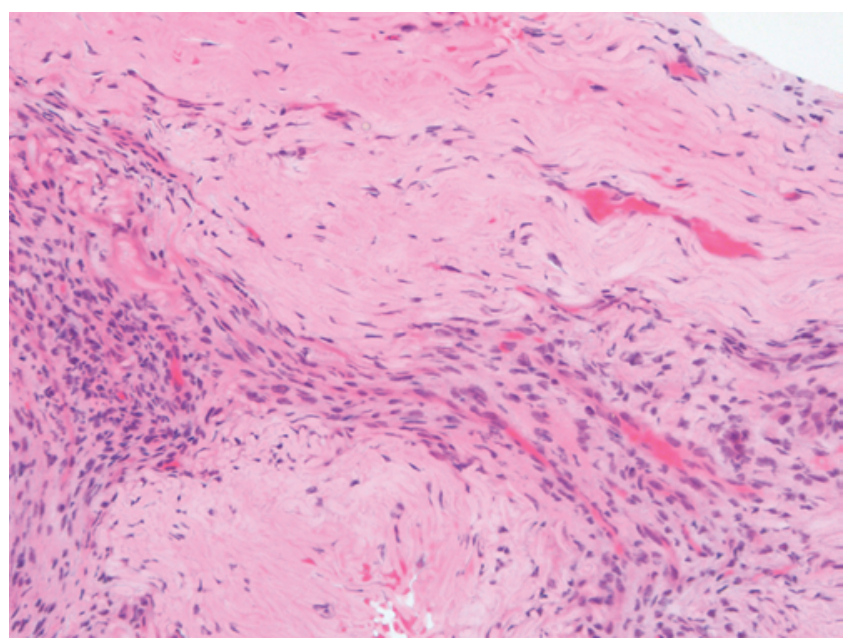

FIG. 5. Photomicrograph revealing bone and fibroconnective tissue with reactive changes, predominantly a large number of macrophages and reactive hyperplasia of the stroma. $H \& E$, original magnification $\times 200$. Figure is available in color online only. 
ings were concerning for discitis and epidural abscess in the form of skip lesions including both the thoracic and cervical spine.

Grobost et al. reported the first case of lumbar pseudogout presenting with radiographic findings concerning for spondylodiscitis and epidural abscess. ${ }^{8}$ The patient was an 85-year-old man with prostate cancer in remission who presented with acute low-back pain; imaging findings were concerning for spondylodiscitis at L2-3. Blood cultures were sterile, and 2 separate percutaneous needle biopsies of the vertebrae were unremarkable for organism growth or metastatic disease. During the patient's hospital course, he developed knee symptoms concerning for pseudogout, and synovial fluid examination revealed CPPD. $\mathrm{He}$ was treated with long-term colchicine and returned 4 years later with low-back and leg pain. Spine MRI demonstrated what appeared to be spondylodiscitis at L4-5 with an associated lumbar epidural abscess. Blood cultures and percutaneous lumbar needle biopsy samples were again sterile, and pathological examination showed signs consistent with calcium pyrophosphate crystals. The patient was treated with colchicine; he experienced symptomatic improvement within 3 days and normalization of inflammatory markers within 1 week.

The pathogenesis of pseudogout requires formation of calcium pyrophosphate crystals in the pericellular matrix of cartilage. ${ }^{25}$ Once generated, these deposits induce inflammation but also have direct catabolic effects on chondrocytes and synoviocytes, resulting in production of metalloproteinases and prostaglandins that can destroy the pericellular matrix.13,22,25 The calcium crystals additionally affect the mechanical properties of the cartilage and may accelerate joint damage. ${ }^{18,25}$

The disease can be sporadic or associated with metabolic disease, trauma, or surgical procedures. ${ }^{16,24}$ Well-known associated metabolic conditions include those that result in a high ratio of inorganic pyrophosphate to phosphate ions, such as in patients with hypophosphatasia. ${ }^{25}$ Chronic hypomagnesemia due to Gitelman's and Bartter's syndromes has also been associated with acute pseudogout.$^{14}$ Calcium metabolic derangements after parathyroidectomy may also result in CPPD. ${ }^{3,24}$ The surgery produces rapid decreases of calcium in the setting of prior hypercalcemia, subsequently inducing shedding of crystals into the synovial fluid. ${ }^{29}$ In the setting of hemochromatosis or iron overload, iron accumulation within joint tissues can result in CPPD. ${ }^{14}$ Other conditions associated with CPPD include hypothyroidism, neuropathic joints, and amyloidosis. ${ }^{26}$

As briefly mentioned, surgery has been linked to acute CPPD flairs. Acute crystal arthritis (including both gout and pseudogout) has been diagnosed in knees and hips after surgical procedures of those respective joints. ${ }^{1,4,9-12}$, ${ }^{30,31}$ In 2012, Ogawa et al. reported the first case of acute lumbar spinal pseudogout following an instrumented lumbar surgery. ${ }^{20}$ CPPD was detected within fluid that had accumulated in the posterior back muscles. That same year, Odate et al. reported 6 cases of adjacent-segment CPPD deposition following thoracolumbopelvic fixation. ${ }^{19}$ In all cases, the CPPD involvement was within or immediately adjacent to the surgical site, diagnosed by identification of CPPD and multinucleated giant cells within the liga- mentum flavum removed during the subsequent decompressive laminectomies. In contrast to these reports, in the present case the patient developed pseudogout at levels remote from the lumbar fusion, involving both cervical and thoracic elements.

Diagnosis is definitive when CPPD crystals are identified by polarized light microscopy in the synovial fluid of affected joints. ${ }^{16}$ The deposits appear as positively birefringent rhomboid-shaped crystals. ${ }^{25}$ However, a caveat exists in the preparation of the tissue for pathological examination, which requires decalcification processing that can dissolve the CPPD crystals, making diagnosis difficult. ${ }^{15,32}$ In the present case, pathology revealed bone and fibroconnective tissue with a large number of macrophages and reactive hyperplasia of the stroma. At the time this specimen was obtained, pseudogout was not considered as a potential diagnosis by the surgical or pathology team. As a result, special processing was not performed at that time. Subsequently, left ankle joint aspiration days later led the rheumatologist to identify objects suspicious for calcium pyrophosphate deposition.

Treatment of pseudogout involves reduction of inflammation and often includes intraarticular glucocorticoids, systemic glucocorticoids, oral colchicine, and nonsteroidal antiinflammatory drugs. ${ }^{14}$ Drugs targeting interleukin-1, such as anakinra, offer considerable potential as well, and this medication was proven successful in the case we report. ${ }^{17}$

Overall, the case reported here demonstrates a unique presentation of pseudogout involving skip lesions of the cervical and thoracic spine after a lumbar fusion. Furthermore, radiographic findings were consistent with discitis and epidural abscess; this is the second report to present such a finding. We encourage spine surgeons to keep pseudogout in mind should a patient present with similar clinical and radiographic findings in the setting of sterile biopsies. A high index of suspicion for pseudogout needs to be maintained in patients who have spinal fusions and develop back pain with high inflammatory markers in the postoperative setting. Since pseudogout is a crystal arthritis, it can develop in segments adjacent to fixated areas, other sites in the spine, or distant joints throughout the body. A request of the pathology team to assess for calcium pyrophosphate crystals may provide a greater chance of successful diagnosis, and early rheumatological consultation may prove beneficial for early symptom management.

\section{Conclusions}

We report a unique case of pseudogout involving skip lesions of the cervical and thoracic spine after a lumbar fusion. The radiographic and clinical picture in this case was suspicious for discitis and spinal epidural abscess, but with repeated sterile biopsies (percutaneous and open) and blood cultures. Once a CPPD diagnosis was confirmed from ankle joint aspiration results, the patient was treated with antiinflammatory medication, with immediate resolution of symptoms. A potential diagnosis of spinal pseudogout should be considered in this unique setting when biopsy samples remain sterile. 


\section{Acknowledgments}

We thank Shirley McCartney, PhD, and Andy Rekito, MS, for editorial and illustrative assistance, respectively. We additionally thank Aaron Halfpenny, DO, for providing the pathology figure.

\section{References}

1. Archibeck MJ, Rosenberg AG, Sheinkop MB, Berger RA, Jacobs JJ: Gout-induced arthropathy after total knee arthroplasty: a report of two cases. Clin Orthop Relat Res (392):377-382, 2001

2. Bencardino JT, Hassankhani A: Calcium pyrophosphate dihydrate crystal deposition disease. Semin Musculoskelet Radiol 7:175-185, 2003

3. Bilezikian JP, Connor TB, Aptekar R, Freijanes J, Aurbach GD, Pachas WN, et al: Pseudogout after parathyroidectomy. Lancet 1:445-446, 1973

4. Blyth P, Pai VS: Recurrence of gout after total knee arthroplasty. J Arthroplasty 14:380-382, 1999

5. Dieppe PA, Alexander GJ, Jones HE, Doherty M, Scott DG, Manhire A, et al: Pyrophosphate arthropathy: a clinical and radiological study of 105 cases. Ann Rheum Dis 41:371376, 1982

6. Felson DT, Naimark A, Anderson J, Kazis L, Castelli W, Meenan RF: The prevalence of knee osteoarthritis in the elderly. The Framingham Osteoarthritis Study. Arthritis Rheum 30:914-918, 1987

7. Ferrone C, Andracco R, Cimmino MA: Calcium pyrophosphate deposition disease: clinical manifestations. Reumatismo 63:246-252, 2012

8. Grobost V, Vayssade M, Roche A, Kemeny JL, Soubrier M: Axial calcium pyrophosphate dihydrate deposition disease revealed by recurrent sterile spondylodiscitis and epidural abscess. Joint Bone Spine 81:180-182, 2014

9. Harato K, Yoshida H: Pseudogout in the early postoperative period after total knee arthroplasty. J Arthroplasty 28:374. e9-374.e11, 2013

10. Healey JH, Dines D, Hershon S: Painful synovitis secondary to gout in the area of a prosthetic hip joint. A case report. $\mathbf{J}$ Bone Joint Surg Am 66:610-611, 1984

11. Holt G, Vass C, Kumar CS: Acute crystal arthritis mimicking infection after total knee arthroplasty. BMJ 331:13221323,2005

12. Kobayashi H, Akizuki S, Takizawa T, Yasukawa Y, Kitahara J: Three cases of pseudogout complicated with unicondylar knee arthroplasty. Arch Orthop Trauma Surg 122:469471, 2002

13. Liu-Bryan R, Pritzker K, Firestein GS, Terkeltaub R: TLR2 signaling in chondrocytes drives calcium pyrophosphate dihydrate and monosodium urate crystal-induced nitric oxide generation. J Immunol 174:5016-5023, 2005

14. Macmullan P, McCarthy G: Treatment and management of pseudogout: insights for the clinician. Ther Adv Musculoskelet Dis 4:121-131, 2012

15. Manhas A, Kelkar P, Keen J, Rostad S, Delashaw JB: Recurrent craniocervical pseudogout: indications for surgical resection, surveillance imaging, and craniocervical fixation. Cureus 8:e511, 2016

16. McCarthy G: Calcium pyrophosphate dihydrate, hydroxyapatite, and miscellaneous crystals, in Klippel JH, Stone JH, Crofford LJ, et al (eds): Primer on the Rheumatic Diseases. New York: Springer, 2008, pp 263-270

17. McGonagle D, Tan AL, Madden J, Emery P, McDermott MF: Successful treatment of resistant pseudogout with anakinra. Arthritis Rheum 58:631-633, 2008

18. Muehleman C, Li J, Aigner T, Rappoport L, Mattson E, Hirschmugl C, et al: Association between crystals and cartilage degeneration in the ankle. J Rheumatol 35:1108-1117, 2008
19. Odate S, Shikata J, Fujibayashi S, Hosaka N, Soeda T, Kimura H: Progressive thoracic myelopathy caused by spinal calcium pyrophosphate crystal deposition because of proximal junctional vertebral compression fracture after lumbopelvic fusion. Eur Spine J 21:2436-2442, 2012

20. Ogawa Y, Nagatsuma M, Kubota G, Inoue G, Eguchi Y, Orita S, et al: Acute lumbar spinal pseudogout attack after instrumented surgery. Spine (Phila Pa 1976) 37:E1529-E1533, 2012

21. Ranschaert E: CPPD of the cervical spine. Radiopaedia.org. (http://radiopaedia.org/cases/cppd-of-the-cervical-spine) [Accessed February 14, 2017]

22. Reuben PM, Wenger L, Cruz M, Cheung HS: Induction of matrix metalloproteinase- 8 in human fibroblasts by basic calcium phosphate and calcium pyrophosphate dihydrate crystals: effect of phosphocitrate. Connect Tissue Res 42:1-12, 2001

23. Rosen T, Furman J: Acute calcium pyrophosphate deposition arthropathy. JAAPA 29:1-3, 2016

24. Rosenthal A, Ryan L, McCarty D: Calcium pyrophosphate deposition disease, pseudogout, and articular chondrocalcinosis, in Koopman WJ, Moreland LW (eds): Arthritis and Allied Conditions, ed 15. Philadelphia: Lippincott Williams \& Wilkins, 2005, pp 2373-2396

25. Rosenthal AK, Ryan LM: Calcium pyrophosphate deposition disease. N Engl J Med 374:2575-2584, 2016

26. Ryan L, McCarty DJ: Calcium pyrophosphate crystal deposition disease; pseudogout; articular chondrocalcinosis, in McCarty DJ (ed): Arthritis and Allied Conditions: A Textbook of Rheumatology, ed 10. Philadelphia: Lea \& Febiger, 1985, pp 1515-1546

27. Salcman M, Khan A, Symonds DA: Calcium pyrophosphate arthropathy of the spine: case report and review of the literature. Neurosurgery 34:915-918, 1994

28. Wells CR, Morgello S, DiCarlo E: Cervical myelopathy due to calcium pyrophosphate dihydrate deposition disease. J Neurol Neurosurg Psychiatry 54:658-659, 1991

29. White JC, Brandt FB, Geelhoed GW: Acute pseudogout following parathyroidectomy. Am Surg 54:506-509, 1988

30. Williamson SC, Roger DJ, Petrera P, Glockner F: Acute gouty arthropathy after total knee arthroplasty. A case report. J Bone Joint Surg Am 76:126-128, 1994

31. Zaman M, Sabir N, Mills SP, Charalambous CP: Pseudogout: a rare cause of acute arthritis following arthroscopic anterior cruciate ligament reconstruction. Knee Surg Relat Res 27:194-196, 2015

32. Zünkeler B, Schelper R, Menezes AH: Periodontoid calcium pyrophosphate dihydrate deposition disease: "pseudogout" mass lesions of the craniocervical junction. J Neurosurg 85:803-809, 1996

\section{Disclosures}

The authors report no conflict of interest concerning the materials or methods used in this study or the findings specified in this paper.

\section{Author Contributions}

Conception and design: Than. Acquisition of data: Than, Bridges, Wanchu. Analysis and interpretation of data: all authors. Drafting the article: all authors. Critically revising the article: all authors. Reviewed submitted version of manuscript: Than. Approved the final version of the manuscript on behalf of all authors: Than, Bridges.

\section{Correspondence}

Khoi D. Than, Department of Neurological Surgery, Mail Code: CH8N, Oregon Health \& Science University, 3303 SW Bond Ave., Portland, OR 97239. email: thank@ohsu.edu. 